\title{
Auxologic parameters and response to 2-year therapy with recombinant human growth hormone in growth hormone deficient children with an ectopic posterior pituitary
}

\author{
Maria Kalina,, ${ }^{1}$ Barbara Kalina-Faska, ${ }^{1}$ Katarzyna Gruszczyńska, ${ }^{2}$ Jan Baron, ${ }^{2}$ Anita Cisowska, ${ }^{1}$ \\ Karolina Morawiec, ${ }^{1}$ Paulina Biel-Lewandowska, ${ }^{1}$ Ewa Małecka-Tendera ${ }^{1}$
}

${ }^{1}$ School of Medicine in Katowice, Department of Paediatrics, Paediatric Endocrinology and Diabetes, ${ }^{2}$ School of Medicine in Katowice, Department of Radiology and Nuclear Medicine; Medical University of Silesia, Katowice, Poland

\begin{abstract}
BACKGROUND: Structural defects of the hypothalamic-pituitary area in MRI are suggested as being a more accurate marker of growth hormone deficiency (GHD) than laboratory assays. OBJECTIVE: To compare auxological characteristics in GHD children with normal pituitary (NP) function and with ectopic posterior pituitary (EPP), prior to therapy with recombinant human growth hormone (rhGH), extending the follow-up to two years following treatment. DESIGN: Eighty-six (86) GHD patients were divided into two groups depending on the pituitary MRI: the EPP (23 children, 3.2-16.8 years old) and the NP group (63 children, 3.3-14.8 years old). Height deficits in the population (hSD) and parents (hSD-mpSD) and the change of hSD and bone/chronological age ratio were assessed before and after 12 and 24 months of rhGH therapy. RESULTS: Height deficits before treatment were significantly greater in EPP compared to NP [median $\mathbf{- 4 . 0 7}(-\mathbf{7 . 0 6}, \mathbf{- 2 . 7 5}) v s \mathbf{- 3 . 1 5}(-\mathbf{4 . 9}, \mathbf{- 2 . 3 5})$ for hSD, and $\mathbf{- 3 . 6 5}(\mathbf{- 7 . 0 6}, \mathbf{- 1 . 2 1})$ $v s-1.83(-4.31,-0.28)$ for $\mathbf{h S D}-m p S D ; \mathbf{p}<0.05]$. Bone age was significantly delayed in the EPP group [0.62 $(0.27,0.92)$ vs $0.75(0.21,0.71) ; \mathbf{p}<0.05]$; differences remained significant during follow-up. After 12 months of rhGH therapy, EPP showed significantly greater catch-up growth compared to NP $[\Delta \mathrm{hSD}=1.2(0.42,2.69)$ vs $0.74(0.05,1.48) ; \mathbf{p}<0.05]$. In the $2^{\text {nd }}$ year, height velocity slowed down and was comparable in the two groups. At the conclusion of the study, hSD was similar in both groups, but hSD-mpSD was more deviated in EPP $[-1.79(-3.71,-1.21)$ vs $-1.1(0.98,-0.07) ; p<0.05]$. CONCLUSIONS: The study showed relevant auxologic differences between EPP and NP children, as well as beneficial effects of rhGH therapy in both groups.
\end{abstract}

Key words: Children, Ectopic posterior pituitary, Recombinant growth hormone

Address for correspondence:

Maria Kalina, Department of Paediatrics, Paediatric

Endocrinology and Diabetes, Medical University of Silesia,

ul. Medyków 16, 40-752 Katowice, Poland;

Tel.: +48 32 2071654, Fax: +48 32 2071653,

E-mail: majak_2001@yahoo.com

Received 03-11-2014, Accepted 07-04-2015

\section{INTRODUCTION}

The synthesis of recombinant human growth hormone (rhGH) in 1981 significantly improved therapy of various growth disorders. ${ }^{1,2}$ Growth hormone deficiency (GHD) is the primary indication for rhGH therapy; 
however, even this entity is characterized by significant phenotypic diversity, while variable therapeutic efficacy can be observed. Diagnostic criteria for GHD, which could facilitate optimization of the treatment, remain disputable, often being arbitrary. An increasing number of reports suggest that magnetic resonance imaging (MRI) of the hypothalamic-pituitary area could be a more accurate marker of the deficiency of tropic hormones as compared with laboratory findings. ${ }^{3-5}$ Pituitary stalk interruption syndrome (PSIS), visualised by MRI, is one of several specific congenital midline defects and is considered to be indicative of combined pituitary deficiency. It is defined as a triad consisting of a very thin or interrupted pituitary stalk, an ectopic posterior pituitary (EPP) and hypoplasia or aplasia of the anterior pituitary. In some patients the abnormality may be limited to EPP (also called ectopic neurohypophysis) or to the interrupted pituitary stalk. ${ }^{6}$ Regardless of the cause of GHD, acceleration of height velocity in the first year of rhGH therapy can be observed in a significant number of patients. ${ }^{7}$ However, due to the inconvenience and considerable costs of long-term treatment, it is justified to identify a group of patients in whom the therapeutic effects will also be maintained in the subsequent years. The aim of this study was to compare auxologic indices in GHD children with a normal pituitary image and in GHD patients with ectopic posterior pituitary prior to rhGH therapy and extending the follow-up to two years of treatment.

\section{SUBJECTS AND METHODS}

The two-year follow-up included 86 children (46 boys and 40 girls, aged from 3.2 to 16.8 years with a median age of 10.2 years) who started rhGH therapy on the grounds of auxological criteria (short stature, slow height velocity 6 months prior to the treatment) and laboratory criteria (concentrations of GH in two stimulation tests below $10 \mathrm{ng} / \mathrm{ml}$ ). The treatment was carried out in the Department of Paediatrics, Paediatric Endocrinology and Diabetes of the Medical University of Silesia, Katowice, Poland, during the period of 2001-2010. It was conducted according to the guidelines of the National Growth Hormone Coordination Board.

Patients were divided into two groups depending on the MRI of the hypothalamic-pituitary area. The first group (EPP) comprised 23 children (14 boys and 9 girls), aged 3.2-16.8 years (median age 9.5 years) with an ectopic posterior pituitary. The other group included 63 children (32 boys and 31 girls), aged 3.3-14.8 years (median age 10.2 years), exhibiting a normal imaging appearance of the pituitary (NP). Chronological age was comparable in the two groups and all children were prepubertal (Tanner stage I) at the time of diagnosis and at the start of rhGH therapy. The mean dose of rhGH applied in the study was 0.6 units $(0.2 \mathrm{mg})$ per $\mathrm{kg}$ body mass, per week in both groups.

Exclusion criteria included dysmorphic syndromes, chromosomal aberrations, suspected or confirmed bone dysplasia, low birth weight (children born small for gestational age, SGA) and features of intrauterine growth retardation, chronic systemic diseases and acquired GHD.

Auxological parameters were assessed at the following time intervals: prior to rhGH therapy (0), after 12 months (1) and after 24 months of rhGH therapy (2). The number of standard deviations from the mean population height (hSD), the difference of hSD from the mid-parental height (hSD-mpSD), the change of hSD after 12 and 24 months of rhGH therapy $(\triangle \mathrm{hSD})$ as well as the bone age delay index (ratio of the bone age to the chronological age; BA/CA) were calculated. Height measurements were based on the Polish national growth charts ${ }^{8}$ and bone age was assessed according to the radiological standards by Greulich-Pyle. ${ }^{9}$

Patients' parents/guardians and patients over 16 years of age gave informed consent for participation in the diagnostic and treatment procedures. The study was approved by the Ethics Committee of the Medical University of Silesia, Poland.

\section{STATISTICAL ANALYSIS}

Normality of the variables distribution was checked by the parametric Lilliefors test. The homogeneity of variances was verified by Barlett's test. Comparative analysis of continuous random variables for independent samples in the normal distribution was performed using the ANOVA test and $t$ test. In the event of non- 
Gaussian distribution, the Kruskal-Wallis ANOVA and Mann-Whitney $U$ tests were used. Comparative analysis of variables at the time intervals was performed using the ANOVA Friedman, Wilcoxon signed-rank test or $t$-Student test. Results of the analyses were considered significant when the $p$ level was below 0.05 . Height was converted into number of standard deviations (SDS) from the population mean. Results are expressed as median and ranges, these descriptive statistics being independent of the type of distribution.

\section{RESULTS}

\section{Auxological parameters prior to the rhGH therapy (0)}

At the start of rhGH treatment, the children with
EPP were significantly shorter and presented significantly delayed bone age as compared to the children with normal MRI of the pituitary. Moreover, deviation from the parental height was markedly greater in the EPP group in comparison to the NP group (Table 1). There were no major differences in the median height velocity in the 6-month follow-up period prior to the treatment $(3.9 \mathrm{~cm}$ per year in EPP versus $4.3 \mathrm{~cm}$ per year in NP; $p>0.05$ ) (Table 2).

\section{Auxological parameters after 12 months of rhGH therapy (1)}

During the 12 months of rhGH therapy, considerable improvement of height velocity was observed in both groups as compared to the period before the treatment. However, in the EPP group, height veloc-

Table 1. Comparison of auxologic parameters in patients with EPP ( $n=23)$ and patients with NP ( $n=63)$ before ( 0$)$, after 12 months (1) and 24 months (2) of the therapy with recombinant human growth hormone (rhGH). Results are presented as medians and ranges; *p $<0.05$

\begin{tabular}{|c|c|c|c|}
\hline Period of follow-up & hSD & hSD-mpSD & BA/CA \\
\hline \multicolumn{4}{|l|}{0} \\
\hline$E P P$ & $-3.96 *(-7.06,-2.75)$ & $-3.65 *(-7.06,-1.21)$ & $0.62 *(0.27,0.92)$ \\
\hline$N P$ & $-3.15(-4.9,-2.35)$ & $-1.83(-4.31,-0.28)$ & $0.75(0.21,0.71)$ \\
\hline \multicolumn{4}{|l|}{1} \\
\hline$E P P$ & $-2.76(-4.67,-0.7)$ & $-2.23 *(-5.18,-1.19)$ & $0.66^{*}(0.38,0.93)$ \\
\hline$N P$ & $-2.44(-4.34,-1.33)$ & $-1.11(-2.23,-0.12)$ & $0.81(0.44,0.79)$ \\
\hline \multicolumn{4}{|l|}{2} \\
\hline$E P P$ & $-2.27(-3.3,-0.69)$ & $-1.79 *(-3.71,-1.21)$ & $0.77 *(0.61,0.93)$ \\
\hline$N P$ & $-1.8(-3.58,-0.2)$ & $-1.1(-0.98,-0.07)$ & $0.84(0.79,0.95)$ \\
\hline
\end{tabular}

EPP: ectopic posterior pituitary group; NP: normal pituitary group; hSD: number of standard deviations from the mean population height; hSD-mpSD: the difference of hSD from the mid-parental height; BA/CA: bone age delay index (ratio of the bone age and chronological age).

Table 2. Comparison of height velocity, expressed by the change of hSD before (0), after 12 months (1) and 24 months (2) of therapy with recombinant human growth hormone (rhGH). Results are presented as medians and ranges

\begin{tabular}{lcc}
\hline Period of follow-up & \multicolumn{1}{c}{$\mathbf{h S D}$} & Statistically significant comparisons (p<0.05) \\
\hline $\mathbf{0}$ & $-0.41(-2.11,-0.04)$ & non significant \\
$E P P(\mathrm{~A})$ & $-0.39(-2.2,-0.02)$ & \\
$N P(\mathrm{~B})$ & & \\
$\mathbf{1}$ & $1.2(0.42,2.69)$ & $\mathrm{C} v s \mathrm{~A}, \mathrm{D}$ \\
$E P P(\mathrm{C})$ & $0.74(0.05,1.48)$ & $\mathrm{D} v s \mathrm{~B}, \mathrm{C}$ \\
$N P(\mathrm{D})$ & & \\
$\mathbf{2}$ & & \\
$E P P(\mathrm{E})$ & $0.61(-0.04,1.12)$ & $\mathrm{E} v s \mathrm{~A}, \mathrm{C}$ \\
$N P(\mathrm{~F})$ & $0.56(-0.03,1.2)$ & F vs B, D \\
\hline
\end{tabular}

EPP: ectopic posterior pituitary group; NP: normal pituitary group; $\triangle \mathrm{hSD}$ : change of number of standard deviations from the mean population height. 
ity, as expressed by the change in hSD over the time $(\triangle \mathrm{hSD} 1)$, was significantly greater than in the NP group (Table 2). Bone age remained significantly more delayed in the EPP group by comparison with the NP group (Table 1). After 12 months of treatment, all EPP children remained prepubertal, whereas 20 children in the NP group (31.7\%) entered puberty (Tanner stage II).

\section{Auxological parameters after 24 months of the rhGH therapy (2)}

In the $2^{\text {nd }}$ year of treatment there was no significant difference in $\triangle \mathrm{hSD}$ between the EPP and NP groups. In both groups height velocity decelerated in comparison to the previous year; however, it remained significantly greater in relation to the pre-treatment period (Table 2). No significant differences in the height deficit between the groups were found after 24 months of the rhGH therapy, apart from deviation from the mid-parental height, which was greater in the EPP group as compared to the NP (Table 1). Bone age remained more delayed in the EPP group (Table 1). In total, 6 children in the EPP group manifested early pubertal signs Tanner stage II). Puberty also proceeded in the NP group with another 12 children entering puberty.

\section{DISCUSSION}

The results of this study reveal auxologic differences between GHD patients with structural abnormality in the form of ectopy of the posterior pituitary and subjects with normal MRI of the hypothalamic-pituitary region. The first group manifested significantly greater height deficits in relation to population and familial potential, however achieving sufficient catch-up growth and eventually benefiting from rGH therapy as other GHD patients.

Diagnosis of GHD is a multistage process in which an accurate auxological assessment remains a fundamental criterion for selecting subjects for rhGH therapy. ${ }^{2}$ Biochemical parameters may appear to be highly variable, depending on the arbitrarily acknowledged cut-off values for the GH concentrations defining its deficiency, ranging from 3 to $10 \mathrm{ng} / \mathrm{ml}$, depending on the age and assays. ${ }^{10,11}$ Furthermore, $\mathrm{GH}$ assays are characterized by low reproducibility and changeable specificity and sensitivity. ${ }^{12-14}$ Among auxiliary diagnostics methods, MRI has been gaining an apparent role as an indicator of hormonal deficiencies of the pituitary. ${ }^{4,5}, 15-17$ According to the guidelines of several endocrine societies, GH stimulation tests are not necessary in the event of co-existence of clinical signs of GHD and structural anomalies of the hypothalamic-pituitary area in the MRI. ${ }^{2}$ Pituitary stalk interruption syndrome with ectopia of the posterior pituitary (PSIS), characterized by the hyperintensive T1 signal, is one of the typical qualitative alternations in the MRI related to GHD. ${ }^{16,18}$

Based on the aforementioned reports, the aim of our study was to determine specifically whether patients diagnosed with GHD according to standardized biochemical criteria, varying in terms of the MRI of the hypothalamic-pituitary area, present different auxological characteristics before and during the substitution rhGH therapy. Patients and auxiliary examinations, including MRI, were assessed within one centre. In terms of efficacy of the rhGH treatment, basic auxological parameters which are commonly used by clinicians were evaluated. Analyses of large groups of patients show improvement of height velocity in the first year of treatment in the majority of subjects treated with rhGH.7 In our study, the follow-up was extended up to two years in order to find out if there are differences in efficacy of the therapy between the examined groups also in the further perspective.

Various reports disclose that patients with GHD co-existing with structural anomalies of the hypothalamic-pituitary area differ in terms of auxology from subjects with normal pituitary., ${ }^{4,17}$ According to Coutant et al, children with GHD and abnormal MRI manifest greater height deficit and younger chronological age at initial diagnosis. ${ }^{4}$ Moreover, Maghnie et al found bone age to be more delayed in this group of patients. ${ }^{5}$ By contrast, Zenaty et al reported that auxological indices were comparable in the prepubertal period, regardless of the pituitary image..$^{20}$ In our study, both chronological age and the pubertal stage were similar in the two analyzed groups, while in addition no significant differences between the groups were observed in terms of height velocity in the pre-treatment period. This could be explained by uniform auxological criteria, justifying diagnostics of the short stature, among which slow height velocity is fundamental. Nevertheless, children 
with EPP presented greater height deficit than NP children, as compared to peers and parents. Coutant et al observed that the auxological characteristics of GHD patients with normal MRI of the pituitary is similar to subjects with idiopathic short stature. ${ }^{4}$ This finding could suggest that in the first group, short stature is caused by some extra-hormonal factors, e.g. genetic or familial factors. In our study, deviation from the parental height decreased in both groups during the following months of observation, which was connected with improved height velocity and subsequent equalizing height deficits in the treated children.

Bone age delay was another differential factor, which was significantly greater in the EPP group as compared to the NP group, similarly to the studies by Maghnie et al. ${ }^{5}$ Moreover, significant bone delay was maintained in the following months of the rhGH therapy in the EPP group, but not in the NP group. This could be explained in part by slower or no pubertal progress in the EPP patients, who may manifest combined pituitary hormonal deficiency, including gonadotropins. As mentioned previously, all children were prepubertal at diagnosis; however, nearly one third of the children in the NP group entered spontaneous puberty already in the first year of $\mathrm{rhGH}$ therapy, while the EPP children still did not exhibit any pubertal signs in the first year of observation and only six subjects presented sexual development in following months. In order to determine whether the bone delay conditions prolonged the growing period, resulting in the better final height in the EPP patients, reassessment after cessation of the rhGH therapy should be carried out. ${ }^{21}$ On the other hand, Leger et al also demonstrated that as many as $22 \%$ subjects with EPP and childhood-onset GHD present normal GH secretion after GH withdrawal. ${ }^{21}$ Unfortunately, the number of patients with EPP in our Department who have already reached final height is too small for a reliable analysis.

Analyzing height velocity in respective years, a significantly greater change was observed in the EPP group as compared to the NP group in the first year of treatment, followed by a levelling off of height velocity in both groups. After two years, the height of the EPP children was comparable to that of the children with normal MRI of the pituitary. Children with EPP, due to a greater initial growth deficit, presented more dynamic growth. Maghnie et al proposed that the final height depends on the prepubertal height velocity and on the extent of the height deficit at the start of the rhGH therapy. ${ }^{5}$ Significant improvement of height in the first year of treatment in both groups in our study may be considered as a favorable prognostic factor of adult stature.

As shown in this study, the presence of the midline defects in patients even with severe short stature may appear to be a good prognostic factor of the response to the treatment and enables catch-up of the deficits. It can also be an indication for more stringent monitoring during puberty and vigilance with regard to putative development of other hormonal deficiencies of the hypothalamic-pituitary axis. ${ }^{17,22-27}$ It should be underlined, however, that normal anatomy of the pituitary does not exclude severe GHD and combined pituitary deficiency, as is found in the case of gene mutations of the pituitary transcription factors, POUF $1 F$ and PROP-1. ${ }^{17,28}$

The results of this study demonstrate a beneficial effect of rhGH therapy both in patients with normal structure of the pituitary as well as in patients with midline defects of the hypothalamic-pituitary axis, provided that basic auxological criteria for the treatment are fulfilled. Thus, a careful and accurate auxological assessment, and not hypothalamic-pituitary area morphology itself, remains a fundamental criterion for selecting subjects for rhGH therapy.

\section{REFERENCES}

1. Milner RD, 1990 Proper use of growth hormone. Arch Dis Child 1: 70-71.

2. Growth Hormone Research Society, 2000 Consensus guidelines for the diagnosis and treatment of growth hormone $(\mathrm{GH})$ deficiency in childhood and adolescence: summary statement of the GH Research Society. J Clin Endocrinol Metab 85: 3990-3993.

3. Tillmann V, Tang VW, Price DA, Hughes DG, Wright NB, Clayton PE, 2000 Magnetic resonance imaging of the hypothalamic-pituitary axis in the diagnosis of growth hormone deficiency. J Pediatr Endocrinol Metabol 13: 1577-1583.

4. Coutant R, Rouleau S, Despert F, Magontier N, Loisel D, Limal JM, 2001 Growth and adult height in $\mathrm{GH}$-treated children with nonacquired $\mathrm{GH}$ deficiency 
and idiopathic short stature: the influence of pituitary magnetic resonance imaging findings. J Clin Endocrinol Metab 10: 4649-4654.

5. Maghnie M, Lindberg A, Koltowska-Häggström M, Ranke MB, 2013 Magnetic resonance imaging of CNS in 15,043 children with GH deficiency in KIGS (Pfizer International Growth Database). Eur J Endocrinol 168: 211-217.

6. Grech R, Galvin L, Looby S, O'Hare A, Thornton J, Brennan P, 2013 Teaching neuroimages: ectopic posterior pituitary. Neurology 81: e121-122.

7. Reiter EO, Price DA, Wilton P, Albertsson-Wikland K, Ranke MB, 2006 Effect of growth hormone (GH) treatment on the near-final height of 1258 patients with idiopathic GH deficiency: analysis of a large international database. J Clin Endocrinol Metab 91: 2047-2054.

8. Palczewska J, Niedźwiecka Z, 2001 Somatic development indices in children and youth of Warsaw. Med Wieku Rozwoj 2: Suppl 1: 18-118.

9. Greulich WW, Pyle SI 1959 Radiographic atlas of skeletal development of the hand and wrist, Stanford, California: Stanford University Press.

10. Binder G, 2011 Growth hormone deficiency: new approaches to the diagnosis. Pediatr Endocrinol Rev 9: 535-537.

11. Chaler EA, Ballerini G, Lazzati JM, et al, 2013 Cut-off values of serum growth hormone $(\mathrm{GH})$ in pharmacological stimulation tests (PhT) evaluated in short-statured children using a chemiluminescent immunometric assay (ICMA) calibrated with the International Recombinant Human GH Standard 98/574. Clin Chem Lab Med 51: 95-97.

12. van der Broeck J, Hering P, Van de Lely A, HokkenKoelega A, 1999 Interpretative difficulties with growth hormone provocative retesting in childhood-onset growth hormone deficiency. Horm Res 51: 1-9.

13. Hilczer M, Smyczyńska J, Lewinski A, 2006 Limitations of clinical utility of growth hormone stimulating tests in diagnosing children with short stature. Endocr Regul 40: 69-75.

14. Mauras N, Walton P, Nicar M, Welch S, Rogol AD, 2000 Growth hormone stimulation testing in both short and normal statured children: use of an immunofunctional assay. Pediatr Res 47: 614.

15. Binder G, Nagel BHP, Ranke MB, Mullis PE, 2002 Isolated GH deficiency (IGHD) type II: imaging of the pituitary gland by magnetic resonance reveals characteristic differences in comparison with severe IGHD of unknown origin. Eur J Endocrinol 147: 755-760.

16. Garel C, Léger J, 2007 Contribution of magnetic resonance imaging in non-tumoral hypopituitarism in children. Horm Res 67: 194-202.

17. Deal C, Hasselmann C, Pfäffle RW, et al, 2013 Asso- ciations between pituitary imaging abnormalities and clinical and biochemical phenotypes in children with congenital growth hormone deficiency: data from an international observational study. Horm Res Paediat 79: 283-292.

18. Zee CS, Go JL, Kim PE, Mitchell D, Ahmadi J, 2003 Imaging of the pituitary and parasellar region. Neurosurg Clin N Am 1: 55-80.

19. Tauber M, Chevrel J, Diene G, et al, 2005 Long-term evolution of endocrine disorders and effect of GH therapy in 35 patients with pituitary stalk interruption syndrome. Horm Res 64: 266-273.

20. Zenaty D, Garel C, Limoni C, Czernichow P, Leger J, 2003 Presence of magnetic resonance imagining abnormalities of the hypothalamic-pituitary axis is a significant determinant of the first 3 years growth response to human growth hormone treatment in prepubertal children with nonaquired growth hormone deficiency. Clin Endocrinol (Oxf) 58: 647-652.

21. Léger J, Danner S, Simon D, Garel C, Czernichow P, 2005 Do all patients with childhood-onset growth hormone deficiency (GHD) and ectopic neurohypophysis have persistent GHD in adulthood? J Clin Endocrinol Metab 90: 650-656.

22. Hamilton J, Blaser S, Daneman D, 1998 MR imaging in idiopathic growth hormone deficiency. Am J Neuroradiol 9: 1609-1615.

23. Triulzi F, Scotti G, di Natale B, et al, 1994 Evidence of a congenital midline brain anomaly in pituitary dwarfs: a magnetic resonance imaging study in 101 patients. Pediatrics 93: 409-416.

24. Kornreich L, Horev G, Lazar L, Schwarz M, Sulkes J, Pertzelan A, 1998 MR findings in growth hormone deficiency: correlation with severity of hypopituitarism. AJNR Am J Neuroradiol 19: 1495-1499.

25. El Chehadeh S, Bensignor C, de Monléon JV, Méjean N, Huet F, 2010 The pituitary stalk interruption syndrome: endocrine features and benefits of growth hormone therapy. Ann Endocrinol (Paris) 71: 102-110.

26. Kulkarni C, Moorthy S, Pullara SK, Rajeshkannan R, Unnikrishnan AG, 2012 Pituitary stalk transection syndrome: Comparison of clinico-radiological features in adults and children with review of literature. Indian J Radiol Imaging 22: 182-185.

27. Wang Q, Hu Y, Li G, Sun X, 2014 Pituitary stalk interruption syndrome in 59 children: the value of MRI in assessment of pituitary functions. Eur J Pediatr 173: 589-595.

28. Osorio MGF, Marui S, Jorge ALA, et al, 2002 Pituitary magnetic resonance imagining and function in patients with growth hormone deficiency with and without mutations in GHRH-R, GH-1, or PROP-1 genes. J Clin Endocrinol Metab 87: 5076-5084. 